\title{
An Approach to Detect Sentence Level Sarcasm Using Deep Learning Techniques
}

\author{
Amruta K. Chimote ${ }^{1}$, S.R. Tandan ${ }^{2}$ and Rohit Miri ${ }^{3}$ \\ ${ }^{1}$ Research Scholar, Department of Computer Science \& Engineering, \\ Dr. C.V. Raman University, Bilaspur (CG), India \\ ${ }^{2,3}$ Associate Professor, Department of computer science \& Engineering, \\ Dr. C.V. Raman University, Bilaspur (CG), India
}

\section{ABSTRACT}

Today is the era of customer self-service, where people use conversation agents (chatbots) to get their query solved in minimum time and cost. Use of conversation Agent gives real time experience to user/customer to get answers very fast. To make this experience more genuine it needs understanding of human emotions and which is the most complex task to perform as facial expression and verbal details are unavailable. Most of data over the internet is in textual format which needs to process to get required answers that is why one of the most popular area in natural language processing is Sentiment analysis which focuses on solving this issue. Sarcasm is critical sentiment which is very difficult to recognize by machines. User express sarcasm to show their anger, disagreement using positive words over internet forums, social media and over shopping sites for reviews about product, services, situation, workplace etc. This paper gives a combined approach by extracting pragmatic features like emoticons, use of hyperbole, punctuations and special words used in sentence to detect sentence level sarcasm using deep learning techniques such as LSTM which will help machine for better understanding of natural language.

KEY WORDS: SARCASM, SENTIMENT ANALYSIS, CONVERSATION AGENT, LSTM, NATURAL LANGUAGE UNDERSTANDING.

\section{INTRODUCTION}

Sarcasm is one of the sentiments belongs to anger or disagreement representing something positive with negative intent. Sarcasm is defined by so many people with different approach. Some of definitions are:

a. (Mondher etl.,2016) elaborated Sarcasm as form of irony, majorly used on internet platform such as social media and blogging sites.

\section{ARTICLE INFORMATION}

*Corresponding Author: chopade.amruta@gmail.com Received 11th Oct 2020 Accepted after revision 26th Dec 2020 Print ISSN: 0974-6455 Online ISSN: 2321-4007 CODEN: BBRCBA

Thomson Reuters ISI Web of Science Clarivate Analytics USA and Crossref Indexed Journal

\section{Clarivate Crossef}

NAAS Journal Score 2020 (4.31)

A Society of Science and Nature Publication,

Bhopal India 2020. All rights reserved.

Online Contents Available at: http//www.bbrc.in/

Doi: http://dx.doi.org/10.21786/bbrc/13.14/30 b. Cambridge Dictionary explains sarcasm as remark used to state opposite of what is said, this is to hurt or to criticize in humorous manner. (Cambridge Dictionary, n.d.)

c. Sarcasm is the lowest form of wit, but the highest form of intelligence stated by Oscar Wilde.

d. According to wheel of sentiment Sarcasm is critical expression of anger.

Sentiment analysis specially inclined towards opinion mining is one of the most favoured topics in natural language processing which leads to understand sentiment behind textual data provided by user such as reviews about place, product, person and even situation. Detection of sentiment from text/speech data leads to correct decision making for company. Available methodologies for sentiment analysis find it difficult to process complex sentiments such as sarcasm, rhetorical questions which can affect ability to reach correct decision.

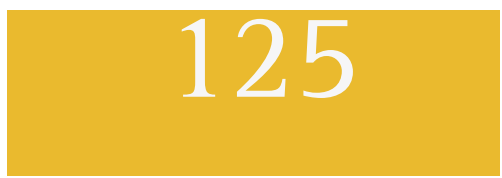


Identification of sarcasm in any text/speech data which may include instructions, blogs, views, or reviews is known as sarcasm detection. Sarcasm detection is emerging area under sentiment analysis and text mining. It is primary need of all conversation Agent to detect sarcasm and generate response based on natural language understanding. Sarcasm detection will be most useful while instructing robots and get efficient work done through it. It further leads to identify the mood, psychology and sometimes even the health condition of people which make human machine interaction more efficient. It is observed that person going through stress or extreme emotion phase starts behaving sarcastic over social media or at workplace, he/she starts giving sarcastic reviews which cannot be identified on textual data by automated system so sarcasm detection can help to identify mental health of person. (Urmila Shrawankar, 2019)

Figure 1: Wheel of Emotions

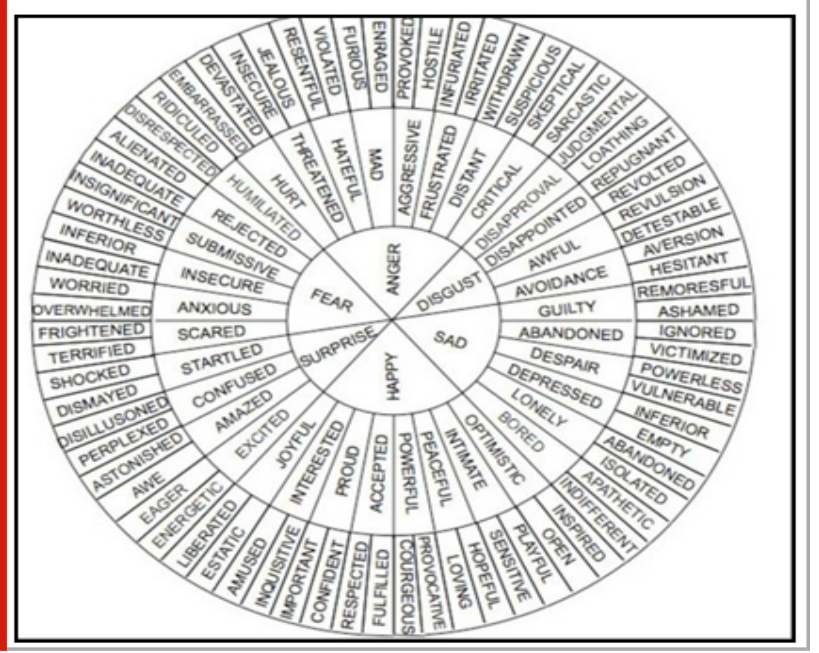

Literature Review: Ample numbers of applications are using sentiment analysis to give user better experience for using chatbots, automated answering machines, etc. Conversation agents are used globally and impacting to reduce overall business cost (Benlian1, march 2020). To make human machine interaction more realistic so many researchers are attracted toward the area of opinion mining and contributed to detection of sarcasm using various approaches. Majorly researchers used social media domain like tweeter and reddit where one can express in minimum number of words so makes it easy for pre-processing. Many researchers used classification, neural network, and deep learning approaches to detect sarcasm.

Initially researchers went with rule-based approach to detect sarcasm, but it is found that use of machine learning and deep learning techniques are more efficient. Rule based approach depends on occurrences. These occurrences are identified, and rules are applied to detect sarcasm. (Kumar, (2017)) Higher accuracy can be achieved by using machine learning techniques. It is observed that extraction of features plays very important role in detecting sarcasm. Research Papers such as Dr. Kalpesh H. Wandra1, 2017 used n-gram (uni,bi,tri) for feature extraction which mainly focuses on pragmatic occurrences in given text used Logistic regression and SVM for classification. In Research work published by (Lakshya Kumar, 2017), steps further for detecting sarcasm and in this author used two approaches where first approach is to get exact noun phrase matching and second approach uses pragmatic feature extraction along with number of tweets embeddings. Here researches got success to get 90\% accuracy by using deep learning methodology.

In scholarly article Encoding Sentiment Information into Word Vectors for Sentiment Analysis (Zhe Ye, August 20-26, 2018 ),primary focus is on following points: (1) The lexicons of sentiment were formed and encoded them into word vectors by FF neural network (2) Fine tuning of Lexicons were done during training of CNN sentiment classifier, (Garg, 2019), in their research paper focused on context of text for that researcher used data from twitter and reddit to detect sarcasm, they used two approaches first one used tf-idf and another one is deep learning approach by using bi directional LSTM and GLoVE. They achieved accuracy of 86\% and 82\% on twitter and reddit datasets, respectively. Sarcasm Detection Using Machine Learning Techniques (P, april 2018), In this paper author used twitter dataset and used multinomial Naïve Bayes to detect sarcasm and used support Vector Machine (SVM) classifier to classify types of sarcasm from tweets.

In paper Sarcasm Detection Building a contextual hierarchy, novel approach is given which is conversation based here author convince about the subjective nature of sarcasm. Author used social platform like twitter and focuses on relation between speaker and listener. Here methodologies used are lexical and pragmatic analyses. (Taradheesh Bali, December 12 2016). Recent research trying to explore sarcasm with new intent like number incongruity in sarcasm detection, rhetorical questions in detecting sarcasm (Aditya Joshi1, 2015) (Abhijeet Dubey, 2019). Some of medical science research papers showcased on detection of brain injuries based on language spoken, detection of sarcasm can help medical science to detect any brain injuries at early stage and autistic nature of person. This may also help in linguistic development. (Autism as well) by (Rambam, 2005) (Channon S) (Katherine P. Rankin, 2009 Oct 1).

\section{MATERIAL AND METHODS}

With the increase in use of online platforms it is mandatory to have human machine interaction in more natural way. Now maximum portal uses automated answering systems, chatbots to answer frequently asked questions by user so require robust methodology to understand human nature and generate response accordingly. In this proposed methodology we use data collection, data pre-processing, feature identification, classification and finally classified text as sarcastic or non-sarcastic. 
Figure 2: Methodology for sarcasm detectio

\begin{tabular}{|c|c|c|c|c|c|}
\hline $\begin{array}{c}\text { Data } \\
\text { Collection }\end{array}$ & $\begin{array}{l}\text { Data from } \\
\text { Twitter }\end{array}$ & & & & \\
\hline $\begin{array}{l}\text { Data Pre- } \\
\text { processing }\end{array}$ & $\begin{array}{c}\text { Data } \\
\text { Cleaning(noise } \\
\text { removal) }\end{array}$ & & $\begin{array}{l}\text { Removal of } \\
\text { shortforms } \\
\text { (SLANGS) }\end{array}$ & Tokenisation & $\begin{array}{c}\text { n ramm } \\
\text { conversion }\end{array}$ \\
\hline $\begin{array}{c}\text { Feature } \\
\text { Identification }\end{array}$ & $\begin{array}{c}\text { pOS } \\
\text { Tagging }\end{array}$ & $\begin{array}{l}\text { Hash } \\
\text { Tags }\end{array}$ & Emoticons & Punctuations & $\begin{array}{l}\text { Repeatition of } \\
\text { character }\end{array}$ \\
\hline Classification & $\begin{array}{l}\text { ML classifiers } \\
\text { of LSTM }\end{array}$ & 1 and use & & & \\
\hline Output & & & & & \\
\hline
\end{tabular}

Long Short-Term Memory is dedicated form of artificial recurrent neural network commonly known as RNN. It has capacity to learn order dependence where prediction is needed. This made it eligible to solve complex domain problem like machine translation, speech recognition etc. Its main focus is to remove problem like vanishing gradient which was present in other versions. LSTM is able to deal with issues like noise, distributed representations and time lags. In LSTM, it's not mandatory to have finite states beforehand. Without fine adjustments LSTM can work with more parameters like rate of learning, handling biases of input and output. LSTM success is reduced complexity up to $\mathrm{O}(1)$. (aditianu, n.d.)

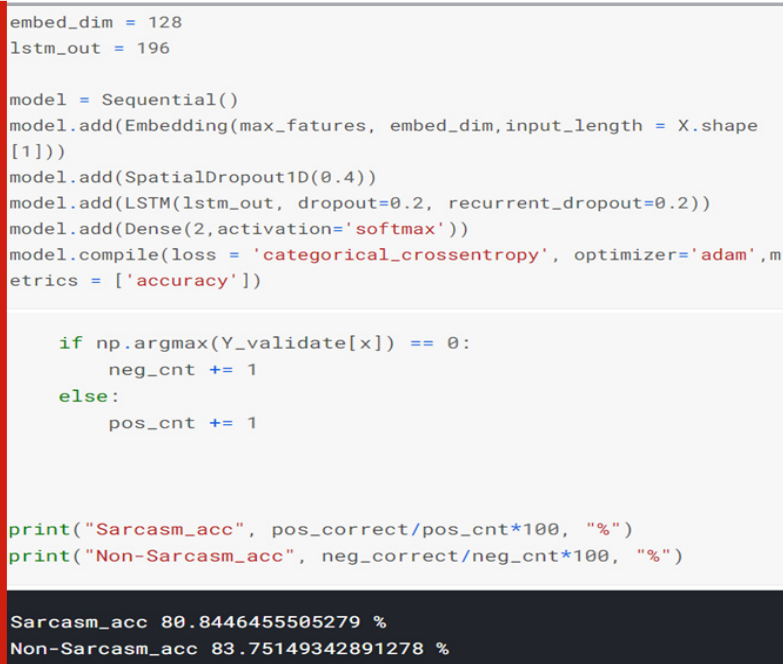

\section{RESULTS}

Partial prototyping model after applying LSTM over political headlines and tweets generated following output. Proposed model correctly classified sarcastic and non-sarcastic sentences with accuracy $80.84 \%$ and $83.75 \%$ respectively.

\section{CONCLUSION}

With the reviewed papers, articles, and applications it is observed that detection of sarcasm is complex. As sarcasm is critical expression of anger one needs to identify the context of sentence before classification. Pragmatic features help to detect sarcasm correctly and use of LSTM increase the accuracy when used with $n$ grams. Scope of this can be extended to identify the incongruity of numbers and autosuggestions before entering sarcastic sentence which will ease the work of machine to respond.

\section{REFERENCES}

Abhijeet Dubey, L. K. (2019),“When Numbers Matter!” Detecting Sarcasm in Numerical Portions of. Proceedings of the 10th Workshop on Computational Approaches to Subjectivity, Sentiment and Social Media Analysis (pp. 72-80), Minneapolis: Association for Computational Linguistics.

Aditianu. (n.d.). Understanding of LSTM Networks. Retrieved from Geeks for Geeks: https://www. geeksforgeeks.org/understanding-of-lstm-networks/

Aditya Joshi1, 2. V. (2015)," Harnessing Context Incongruity for Sarcasm Detection”, Proceedings of the 53rd Annual Meeting of the Association for Computational Linguistics (Pg. 757-762). Beijing, China, July 26-31: Computational Linguistics.

Benlian1, M. A. (March 2020),"AI-based chatbots in customer service and their effects", Springer (https:// doi.org/10.1007/s12525-020-00414-7), 1-19.

Cambridge Dictionary. (n.d.). Retrieved from Cambridge Dictionary: https://dictionary.cambridge.org/dictionary/ english/sarcasm

Channon S, P. A. (n.d.), "Social cognition after head injury: sarcasm and theory of mind"

Dr. Kalpesh H. Wandra1, M. B. (2017), "Sarcasm Detection in Sentiment Analysis”, International Journal of Current Engineering and Scientific Research (IJCESR), 24-32.

Garg, A. K. (2019), "Empirical study of shallow and deep learning models for sarcasm detection using context in benchmark datasets", Journal of Ambient Intelligence and Humanized Computing (2019), Springer.

Katherine P. Rankin, A. S.-T. (2009 0ct 1), "Detecting Sarcasm from Paralinguistic Cues: Anatomic and Cognitive Correlates in Neurodegenerative Disease”, Published in final edited form as: Neuro image (doi: 10.1016/j.neuroimage.2009.05.077).

Kumar, L. S. ((2017)). Approaches for Computational Sarcasm Detection: A Survey. ACM CSUR.

Lakshya Kumar, A. S. (2017), "Having 2 hours to write a paper is fun: Detecting Sarcasm in Numerical", Computational Linguistic.

P, R. K. (April 2018). “Sarcasm Detection Using Machine Learning Techniques”, International Journal of Recent Scientific Research, vol.9 issue4 (L), 26368-26372.

Rambam, S. G.-T. (2005), "The Neuro anatomical Basis of Understanding Sarcasm and Its Relationship to Social Cognition”, Medical Centre Neuropsychology Copyright 
2005 by the American Psychological Association 2005, Vol. 19, No. 3,, $288-300$.

Sarcasm Detection: RNN-LSTM. (2019), Retrieved from Kaggle: https://www.kaggle.com/tanumoynandy/ sarcasm-detection-rnn-lstm

Taradheesh Bali, N. S. (December 12 2016), "Sarcasm Detection Building a contextual hierarchy” Proceedings of the Workshop on Computational Modeling of People's Opinions, Personality, and Emotions in Social Media, (pp. 119-127). Osaka, Japan. Urmila Shrawankar, G. R. (2019), "Sarcasm Detection for Workplace Stress Management”, International Journal of Synthetic Emotions.

Zhe Ye, F. L. (August 20-26, 2018), "Encoding Sentiment Information into Word Vectors for Sentiment Analysis”, Proceedings of the 27th International Conference on Computational Linguistics (pp. 997-1007). New Mexico, USA: Computational Linguistics. 